\title{
Brief exercise induces an immediate and a delayed leucocytosis
}

\author{
D. A. McCarthy PhD, M. Grant* MD, M. Marbut ${ }^{\dagger}$ PhD, M. Watling* MRCGP, \\ A. J. Wade ${ }^{\dagger}$ PhD, I. Macdonald ${ }^{\S}$ PhD, S. Nicholson** MD, R. D. Melsom ${ }^{t+}$ and \\ J. D. Perry ${ }^{\text {tt }}$ FRCP \\ School of Biological Sciences, Queen Mary and Westfield College, London, UK \\ ${ }^{*}$ Sports Medicine, ${ }^{+}$Department of Physiology, and ${ }^{+t}$ Department of Rheumatology, London Hospital and \\ Medical College, London, UK \\ \$Department of Physiology, University of Nottingham, Nottingham, UK \\ **Department of Obstetrics and Gynaecology, UMDS, St. Thomas' Hospital, London, UK
}

\begin{abstract}
Haematological profiles were measured: (1) before and for $5 \mathrm{~h} 30 \mathrm{~min}$ after $30 \mathrm{~min}$ sports (squash, swimming, jogging); and (2) before, during and for $2 \mathrm{~h} 30 \mathrm{~min}$ after $30 \mathrm{~min}$ cycle ergometry at workloads which required rates of oxygen consumption that were between $48 \%$ and $84 \%$ of maximal. In both instances exercise induced an immediate leucocytosis (owing to rises in both neutrophils and lymphocytes) which subsided rapidly at the finish of exercise and was followed by a delayed neutrophilia of greater magnitude which peaked at approximately $3 \mathrm{~h}$ after the start of exercise. Changes in plasma catecholamines and cortisol recorded during and after exercise (cycle ergometry only) support the hypothesis that the immediate leucocytosis during brief exercise is attributable to elevated catecholamine levels, whereas the delayed neutrophilia is due to raised cortisol levels.
\end{abstract}

Keywords: Haematological indices, plasma biochemicals, sports, cycle ergometry

It is well known that exercise can induce an immediate leucocytosis which persists for as long as the effort is sustained ${ }^{1}$. However, it is less widely appreciated that a brief period of exercise can induce not only an immediate leucocytosis which subsides rapidly after the finish of exercise, but also a delayed prolonged leucocytosis which peaks some hours later. Evidence indicating the start of just such a delayed leucocytosis can be seen in several older studies $^{2-7}$, but the phenomenon has generally attracted little attention and consequently is poorly characterized. Recently, in a brief communication elsewhere, we reported the time course of the delayed leucocytosis elicited by $30 \mathrm{~min}$ jogging, squash and swimming ${ }^{8}$. We now present full details of the changes in the peripheral blood indices seen in

Address for correspondence: Dr D. A. McCarthy, School of Biological Sciences, Queen Mary and Westfield College, Mile End Road, London E1 4NS, UK

(C) 1991 Butterworth-Heinemann Ltd

0306-3674/91/040191-05 a slightly extended version of that study (study A), together with the results of cycle ergometer experiments to investigate the mechanisms and magnitude of the immediate and delayed leucocytosis induced by $30 \mathrm{~min}$ exercise at known workloads (studies $B_{1}$ and $B_{2}$ ).

\section{Subjects, materials and methods}

\section{Study A}

Details of the subjects studied and the protocol used have been described already 8 . Briefly, 11 healthy men, mean(s.d.) age 41.4(7.3) years continued in their normal daily routine for $24 \mathrm{~h}$ before the experiment but avoided strenuous exercise. Between $10.00 \mathrm{~h}$ and $11.00 \mathrm{~h}$ each man exercised for $30 \mathrm{~min}$ at a rate close to the maximum that he could sustain for that duration, by playing squash $(n=4)$, jogging $(n=$ 4) or swimming $(n=3)$. Blood samples taken by venepuncture at $0,30,60,165,270$ and $360 \mathrm{~min}$ and at similar times during a normal working (control) day, were collected into $\mathrm{K}_{2}$ EDTA $\left(1 \mathrm{mg} \mathrm{ml}^{-1}\right)$. For statistical analysis subjects were assigned to one of three categories based on the amount of exercise taken during the 3 months preceding the study - four subjects played no sports and took no vigorous exercise whatsoever, three jogged or swam regularly for between $30 \mathrm{~min}$ and $1 \mathrm{~h} 30 \mathrm{~min}$ per week and four played sports (hockey, squash, cycling and jogging) or did circuit training for more than $2 \mathrm{~h}$ per week.

\section{Study $B_{1}$}

Five healthy men participated in this study, two of whom had also participated in study A. Their physical characteristics (means(s.d.)) were age 42.4(4.9) years; height $1.790(0.058) \mathrm{m}$; weight 75.12(8.16) kg; $\dot{V}_{2} \max 2.729(0.271) 1 \mathrm{~min}^{-1}$. Approximately 1 week before the experiment, the maximal oxygen uptake $\left(\dot{V}_{2 \text { max }}\right)$ of each subject was measured using a computer-interfaced exercise testing 
system ${ }^{9}$. Subjects refrained from vigorous exercise for $24 \mathrm{~h}$ before the experiments and from consuming alcohol for $12 \mathrm{~h}$ and caffeine-containing beverages for $24 \mathrm{~h}$, before and during the experiments. Between $09.30 \mathrm{~h}$ and $10.30 \mathrm{~h}$ a small cannula fitted with a three-way valve and maintained patent by a heparinsaline ( 20 units $\mathrm{ml}^{-1}$ ) lock, was inserted into a vein in the antecubital fossa. Approximately $15 \mathrm{~min}$ later, when the subject was seated on an electronically braked bicycle ergometer (Monark, KEM 3, Cranlea and Company, Birmingham, UK), the first blood sample was taken just before starting exercise. The ECG was monitored continuously with a Reigel (Model 302) cardiac monitor (Riegel and Company, Morden, UK) with 'Node crest' modification, and heart rate was collected by counting square wave pulses on one line of the digital port of the computer. Subjects pedalled at between 60 r.p.m. and 80 r.p.m. at a workload of $50 \mathrm{~W}$ for $1 \mathrm{~min}$, and then at a workload (mean(s.d.) 153(42.3) W) calculated to require an oxygen consumption equivalent to $70 \%$ of $V \mathrm{O}_{2 \max }$ for $30 \mathrm{~min}$. Blood samples were taken at 10,20 and $30 \mathrm{~min}$ while the subjects were exercising and at 45,90 and $180 \mathrm{~min}$ while they were at rest. A portion $(1 \mathrm{ml})$ of each sample was collected into $\mathrm{K}_{2}$ EDTA $\left(1 \mathrm{mg} \mathrm{ml}^{-1}\right)$ for haematological determinations, the remainder $(9 \mathrm{ml})$ was mixed with heparin $(10$ units $\mathrm{ml}^{-1}$ ) and the plasma recovered by centrifuging. Plasma for lactate and cortisol analysis was stored frozen at $-20^{\circ} \mathrm{C}$, but plasma for catecholamine analysis was first mixed $(50: 1 \mathrm{v} / \mathrm{v})$ with aqueous glutathione $(6 \%)$ and ethylenediamine tetra-acetic acid (EDTA) $(9.5 \%)$ adjusted to $\mathrm{pH}$ 7. Plasma samples for lactate analysis were thawed and denatured within $60 \mathrm{~min}$ and lactate concentrations determined within $10 \mathrm{~min}$ by an enzymic procedure using an Analox (Model P-GMT) analyser (Analox, London, UK). Plasma cortisol concentrations were determined by radio-immunoassay. Values quoted for both analytes are the means of duplicate determinations. Adrenaline and noradrenaline concentrations were determined by high-pressure liquid chromatography with amperometric detection; values quoted are for single determinations.

\section{Study $B_{2}$}

One subject (who had also participated in studies A and $B_{1}$ ) exercised according to the protocol described for study $B_{1}$ except that he did so on different occasions (14-21 days apart) at 100, 150 and 200 W.

\section{Common procedures}

Haematological parameters were measured using a Coulter (Model S, Coulter Electronics, Luton, UK) (study A, subjects 1-4) or a Technicon (Model H-1, Technicon Instruments, Basingstoke, UK) (study A, subjects 5-11; studies $B_{1}$ and $B_{2}$ ) automatic blood cell analyser. All subjects had normal peripheral blood profiles except for one subject in study $B_{1}$ who had slightly low total leucocyte and neutrophil counts $\left(4.21 \times 10^{9} \mathrm{l}^{-1}\right.$ and $1.49 \times 10^{9} \mathrm{l}^{-1}$, respectively).
Paired $t$ tests (two-tailed) with Bonferroni's correction were used to examine differences between variables on days with and without exercise (study A) and analysis of variance with Duncan's correction for multiple comparisons was used to compare values during and after exercise with those before exercise. Correlations between variables were sought using Pearson's linear regression (coefficient, $r$ ) and Spearman's non-parametric procedure (coefficient, $\rho$ ). All analyses were done using the 'number crunch' program on a personal computer. Significance was accepted at $P<0.05$. All subjects gave their informed consent for the studies.

\section{Results}

\section{Study A}

The results presented in Figure 1 show that the mean(s.e.m.) rise in the leucocyte count after $30 \mathrm{~min}$ exercise of $117(5) \%(0 \mathrm{~min}$ value $=100 \%)$ was due to increases in both lymphocytes and neutrophils, whereas the delayed rise which peaked at $165 \mathrm{~min}$ (156(14)\%) was attributable solely to an increase in neutrophils.

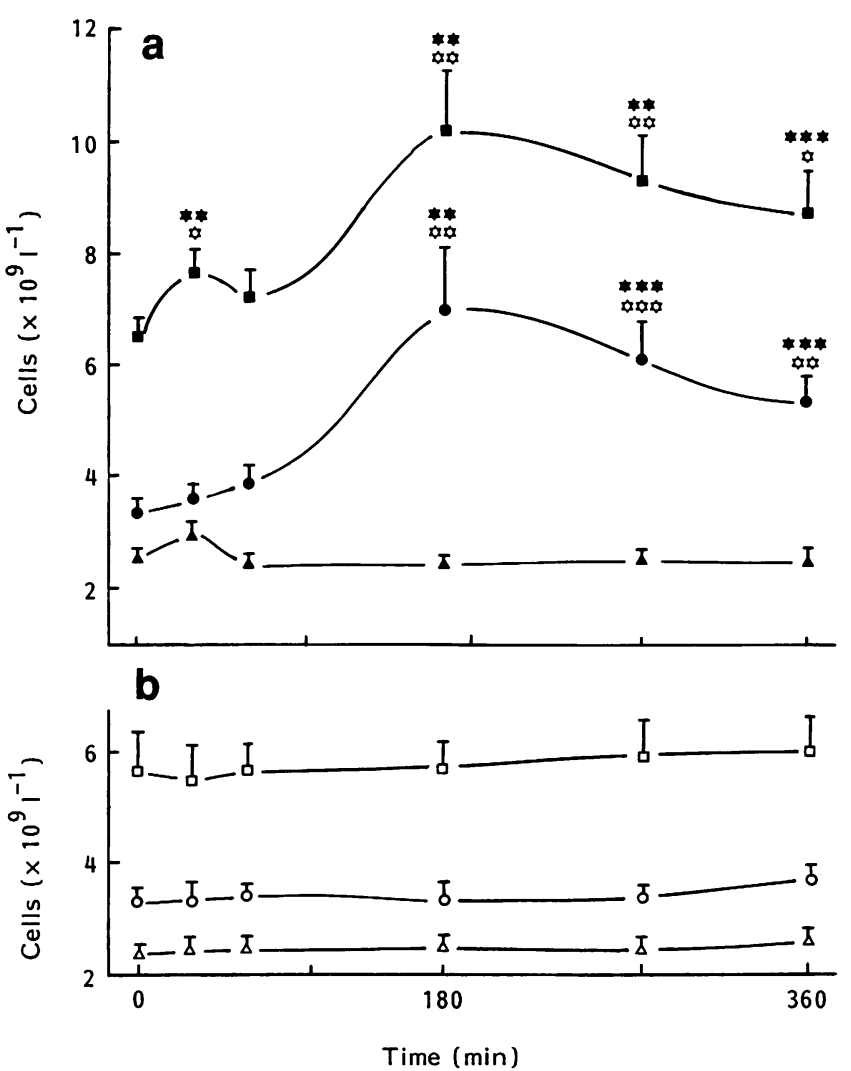

Figure 1. a Effect of $30 \mathrm{~min}$ exercise (between 0 and $30 \mathrm{~min}$ ) on the numbers of: $\square$, circulating leucocytes; $\bullet$, neutrophils; $\Delta$, lymphocytes. Results are the means of 11 experiments (four playing squash, four jogging and three swimming); bars indicate the standard error. Values which differed from those at $0 \mathrm{~min}$ are indicated: $\star \star=P<0.05$; $\star \star \star=P<0.01$; and from those at comparable times on a

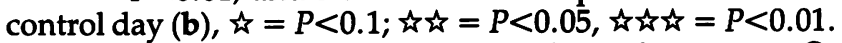
b Variation in the number of: $\square$, circulating leucocytes; $O$, neutrophils; $\Delta$, lymphocytes, in the same subjects during a normal working day without exercise 
The mean leucocyte count immediately after exercise differed from that before exercise but not significantly from that at a comparable time on a control day ( $P<0.1$ only); however, between $3 \mathrm{~h}$ and $6 \mathrm{~h}$ after exercise the mean leucocyte and neutrophil counts differed both from those before exercise and from those at comparable times on a control day.

Considerable intersubject variation which was unrelated to the nature of the exercise occurred: first, in the relative increases in magnitude of both immediate (i.e. at $30 \mathrm{~min}$ ) and delayed leucocytosis; second, in the relative changes in lymphocytes and neutrophils at $30 \mathrm{~min}$; and third, in the time (between 160 and $360 \mathrm{~min}$, when delayed leucocytosis reached a peak (results not shown). Except for an increase in the platelet count $(112.8(2.7) \% ; P<0.01)$ at $30 \mathrm{~min}$, a decrease in haemoglobin concentration $(96.9(0.5) \%$; $P<0.05)$ at $270 \mathrm{~min}$ and decreases in the haematocrit between $30 \mathrm{~min}(97.8(0.5) \%$; n.s.) and $360 \mathrm{~min}$ $(97.0(0.5) \% ; P<0.05)$, there were no significant changes (from the values at $0 \mathrm{~min}$ ) in any of the other haematological parameters measured (monocyte, eosinophil, basophil and erythrocyte numbers, mean corpuscular volume (MCV), mean corpuscular haemoglobin concentration (MCHC) and mean platelet volume (MPV) during the period studied. Blood leucocyte counts at the peak of the delayed leucocytosis were correlated with those at $30 \mathrm{~min}(\rho=$ $0.707, P<0.02 ; r=0.713, P=0.021)$. By $24 \mathrm{~h}$ all haematological parameters (data available for only five experiments) had returned to their pre-exercise levels (results not shown).

\section{Study $B_{1}$}

The effects on the leucocyte count of $30 \mathrm{~min}$ cycle ergometry at $70 \% \quad \dot{V}_{\mathrm{O}_{2} \max }$ (Figure 2) generally resembled those observed previously in study A, except that the magnitude of both immediate $(137(8) \%)$ and delayed (179(19)\%) leucocytosis was greater. Significant changes because of exercise occurred in basophil $(167(20) \%)$, erythrocyte $(107(2) \%)$ and platelet numbers $(122(8) \%)$ at $30 \mathrm{~min}$, and in neutrophil and hence leucocyte numbers at $180 \mathrm{~min}$. At $30 \mathrm{~min}$, lymphocyte numbers were correlated with plasma adrenaline concentrations $(r=0.978, P=0.02 ; \rho=1, P<0.01)$. Neutrophil numbers at $180 \mathrm{~min}$ were correlated with the plasma concentration of cortisol at $30 \mathrm{~min}(r=0.952, P=$ 0.048; $\rho=1, P<0.01$ ).

\section{Study $B_{2}$}

In a preliminary study to assess the effects of relative workload, one subject exercised on different occasions at $48 \%, 61 \%$ and $84 \%$ of his $\dot{V O}_{2 \max }$. The results (Table 1) showed that the percentage changes in plasma lactate, noradrenaline, adrenaline and cortisol concentrations at $30 \mathrm{~min}$ increased progressively with the relative workload. So, too, did the lymphocyte count, but the modest increases in neutrophils at this time (to $114 \%, 130 \%$ and $123 \%$, respectively) were not closely linked to the workload. At $180 \mathrm{~min}$, on the other hand, only the percentage change in the neutrophil count was related progressively to the workload, or to the changes in plasma concentrations of the four metabolic indicators of stress (lactate, noradrenaline, adrenaline and cortisol) as recorded at $30 \mathrm{~min}$.

Table 2 presents results from analysis of five sample times $(0,10,20,30$ and $45 \mathrm{~min})$ during the experiment
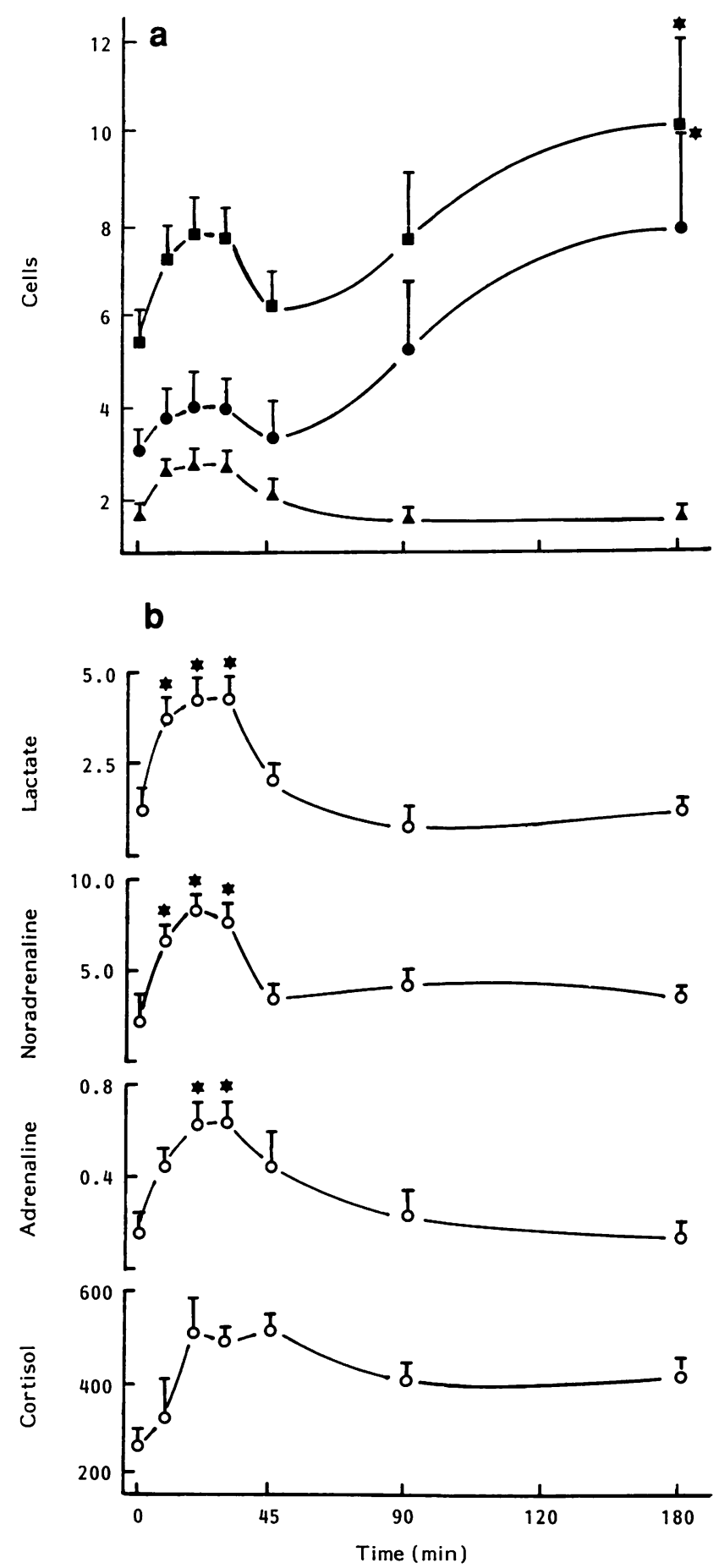

Figure 2. Effect of $30 \mathrm{~min}$ cycle ergometry (between 0 and $30 \mathrm{~min})$ at $70 \% \dot{V}_{\mathrm{O}_{2} \max }$ on a the number of: $\square$, circulating leucocytes; $\bullet$, neutrophils; $\boldsymbol{\Delta}$, lymphocytes. Values are given as $\times 10^{9} 1^{-1}$. b. Effect on the plasma concentration of lactate (as $\mathrm{mM}$ ), noradrenaline (as $\mathrm{nM}$ ), adrenaline (as $\mathrm{nM}$ ) and cortisol (as $\mathrm{nM}$ ). Results are the means of five experiments (study $B_{1}$ ); bars indicate one standard error; values which differ $(P<0.05)$ from those at $0 \mathrm{~min}$ are indicated with an asterisk 
Table 1. Effect of workload on changes in leucocytes and plasma biochemicals during and after exercise*. One subject exercised for 30 min on a cycle ergometer at 100,150 and $200 \mathrm{~W}$ (equivalent, respectively, to $48 \%, 61 \%$ and $84 \%$ of his $\dot{V}_{\mathrm{O}_{2}}$ max ). Values are given as percentages of those at $0 \mathrm{~min}(100 \%)$

\begin{tabular}{|c|c|c|c|c|c|c|}
\hline \multirow[t]{2}{*}{ Parameter } & \multicolumn{3}{|c|}{$30 \min$} & \multicolumn{3}{|c|}{$180 \min$} \\
\hline & $100 \mathrm{~W}$ & $150 W$ & $200 \mathrm{~W}$ & $100 \mathrm{~W}$ & $150 \mathrm{~W}$ & $200 \mathrm{~W}$ \\
\hline Leucocytes & 108 & 140 & 153 & 144 & 142 & 171 \\
\hline Neutrophils & 114 & 130 & 123 & 147 & 161 & 223 \\
\hline Lymphocytes & 76 & 152 & 306 & 97 & 100 & 113 \\
\hline Lactate & 220 & 515 & 990 & 80 & 77 & 90 \\
\hline Noradrenaline & 173 & 357 & 497 & 149 & 176 & 111 \\
\hline Adrenaline & 417 & 600 & 1107 & 117 & 100 & 86 \\
\hline Cortisol & 122 & 213 & 290 & 109 & 115 & 141 \\
\hline
\end{tabular}

*Samples were taken at $0,10,20,30,45,90$ and $180 \mathrm{~min}$, but for simplicity the results for changes at 10, 20, 45 and 90 min have been omitted

Table 2. Correlations between changes in plasma biochemicals and the number of circulating leucocytes in one subject during and after 30 min cycle ergometry at $200 \mathrm{~W}$ (equivalent to $84 \%$ of his $\left.\dot{V}_{\mathrm{O}_{2}, \max }\right)$

\begin{tabular}{lllll}
\hline Cell type & Lactate & Noradrenaline & Adrenaline & Cortisol \\
\hline Leucocytes & 0.9 & 1.0 & 0.9 & 0.2 \\
Neutrophils & 0.82 & 0.98 & 0.82 & 0.05 \\
Lymphocytes & 0.9 & 1.0 & 0.9 & 0.2 \\
\hline
\end{tabular}

Values given are for Spearman's correlation coefficient $(\rho)$ calculated for the five samples taken at $0,10,20,30$ and $45 \mathrm{~min}$. Significant $(P<0.05)$ correlations would be expected where values for $\rho$ exceed 0.9

conducted at a workload of $84 \% \dot{V}_{2}$ max. It is evident that this stressful workload induced changes in both cell counts and some metabolic indicators of stress (lactate, noradrenaline and adrenaline) which were highly correlated, but these associations were not as well defined at lower workloads (results not shown). During exercise the cortisol level rose but the rise was delayed relative to the changes in leucocytes and the more volatile indicators of stress.

\section{Discussion}

Our studies show unequivocally that $30 \mathrm{~min}$ vigorous exercise, either as sports (study A) or as cycle ergometry at $48 \% \dot{V}_{\mathrm{O}_{2} \max }$ to $84 \% \dot{V}_{\mathrm{O}_{2} \max }$ (studies $\mathrm{B}_{1}$ and $B_{2}$ ), causes not only a well recognized immediate transient leucocytosis but also a substantial delayed and prolonged neutrophilia which peaks between 3 and $6 \mathrm{~h}$ later. Similar results were obtained also by Hansen et al. ${ }^{10}$ in a study of the effects of running (between $1.7 \mathrm{~km}$ and $10.5 \mathrm{~km}$ ) and published after this manuscript was submitted. The changes noted in erythrocytes (study $B_{1}$ ) and platelets (studies $A$ and $\left.B_{1}\right)$ are in agreement with those reported previously ${ }^{1-13}$, as are those for the changes in plasma lactate, catecholamine and cortisol levels (studies $B_{1}$ and $\left.B_{2}\right)^{14}$. Since the increase in erythrocyte count is known to be due to the fluid shift from plasma to the tissues, it is reasonable to suppose that up to $7 \%$ of the change in any other cell count may be due to the same cause. Changes greater than this are therefore attributable to cell mobilization.
In some early experiments during study A, light microscopy of stained blood films suggested a 'left shift' might be occurring during delayed neutrophilia. Therefore, subsequent experiments were performed using the Technicon $\mathrm{H}-1$ blood cell analyser, which provides a 'lobularity index' that is related to polymorph nuclear lobe complexity ${ }^{15}$. Marked decreases in the lobularity index during delayed neutrophilia were noted in samples from only two persons (one each from studies $A$ and $B_{1}$, results not shown). Thus an increase in immature cells at this time seems to be a possible, but by no means inevitable, consequence of brief exercise. It should also perhaps be mentioned that the specificity and sensitivity of this parameter under clinical conditions has been questioned ${ }^{16}$. Nevertheless, a moderate 'left shift' was also observed $2 \mathrm{~h}$ after a $10.5 \mathrm{~km}^{\prime}$ run $^{10}$.

Interindividual variation in the magnitude of immediate and delayed leucocytosis seen after sports (study A) was initially thought to be due to differences in the cumulative amount of work done rather than to the intensity of exercise. However, this variability was not markedly reduced when subjects exercised for the same time at $70 \% \dot{V}_{2} \max$ (study $\mathrm{B}_{1}$ ). The reasons for this are not known but may arise from intersubject differences in the production, elimination and effects of stress hormones such as the catecholamines and cortisol, even at identical relative workloads. If correct, one consequence of this might be that correlations between hormone concentrations and leucocyte numbers are more apparent within than between individuals.

The correlation observed between lymphocyte numbers and plasma adrenaline concentrations at the end of exercise (study $B_{1}$ ) has been noted before ${ }^{17}$, but the correlations between leucocyte, neutrophil and lymphocyte numbers and plasma lactate, noradrenaline and adrenaline concentrations found in study $\mathrm{B}_{2}$ (Table 2) have not been reported previously, possibly because they are evident only at high relative workloads. These results provide support for the idea that either noradrenaline or adrenaline, or both, are involved in the immediate mobilization of leucocytes during exercise.

No contemporaneous correlation was found between plasma cortisol levels and leucocyte numbers, either during, or at the finish of $30 \mathrm{~min}$ exercise in 
studies $B_{1}$ or $B_{2}$ which is in complete accordance with earlier reports ${ }^{11,12}$. However, a strong correlation was found in both studies $B_{1}$ and $B_{2}$ between plasma cortisol levels at the end of $30 \mathrm{~min}$ exercise and the magnitude of the delayed neutrophilia at $180 \mathrm{~min}$. It might therefore be argued that a cortisol-mediated mechanism is involved in the delayed but not in the immediate leucocytosis resulting from brief vigorous exercise. It should be noted, however, that these results contradict directly those of Hansen et al. ${ }^{10}$ who found no such correlation, but who reported instead a correlation between cortisol levels and the magnitude of the delayed lymphopenia after brief exercise. The reasons for this discrepancy are not obvious but the relative magnitude of the changes which we observed in cortisol concentrations (up to threefold) was much greater than that reported by those authors.

The biological advantages (if any) of increasing the leucocyte count, either during or after exercise, are not yet understood. However, in terms of its magnitude and duration, delayed leucocytosis seems to be the more important phenomenon and worthy of further investigation. In this respect, our studies have shown that the relative workload and its duration can both affect the response. These results also show that, when investigating the immediate effects of exercise on leucocyte numbers, it is important to study subjects who have refrained from exercise for at least $24 \mathrm{~h}$ to avoid the consequences of a delayed leucocytosis elicited by a previous bout of exercise.

In summary, our results are consistent with, and support, the contention that the immediate leucocytosis and the delayed neutrophilia induced by brief (less than $1 \mathrm{~h}$ ) exercise are due to mechanisms involving increases in the plasma concentrations of catecholamines and cortisol respectively ${ }^{14}$.

\section{Acknowledgements}

This study was supported by a grant from the Nuffield Foundation. We are grateful to Drs I. Harper, J. Camilleri, S. McFarlane and P. Kelly for collecting blood samples (study A), to Professor G.
Jenkins for the use of facilities, and to Mr P. Hall and Mr D. Worby for the haematological analyses.

\section{References}

1 Garrey WE, Bryan WR. Variations in white blood cell counts. Physiol Rev 1935; 15: 597-638.

2 Andersen KL. Leucocyte response to brief, severe exercise. J Appl Physiol 1955; 7: 671-4.

3 Egoroff A. Die veränderung des Blutbildes während der Muskelarbeit bei Gesunden. Z für Klin Med 1924; 100: 485-97.

4 Eskola J, Ruushanen O, Soppi E et al. Effect of sport stress on lymphocyte transformation and antibody formation. Clin Exp Immunol 1978; 32: 339-45.

5 Rohde CP, Wachholder K. Weisses Blutbild und Muskelarbeit. Arbeitsphysiologie 1953; 15: 165-74.

6 Steel CM, Evans J, Smith MA. Physiological variation in circulating B cell:T cell in man. Nature (Lond) 1974; 247: 387-8.

7 Robertson AJ, Padesar KCRB, Potts RC, Gibbs JH, Browning MCK. The effects of strenuous physical exercise on circulating blood lymphocytes and serum cortisol levels. J Clin Lab Immunol 1981; 5: 53-7.

8 McCarthy DA, Perry JD, Melsom RD, Dale MM. Leucocytosis induced by exercise. $\mathrm{Br}$ Med J 1987; 295: 6 .

9 Wade AJ. An exercise testing system designed for student use. J Physiol 1986; 381: $12 \mathrm{P}$.

10 Hansen J-B, Wilsgard L, Osterud B. Biphasic changes in leucocytes induced by strenuous exercise. Eur J Appl Physiol 1991; 62: 157-61.

11 Gimenez M, Mohan-Kumar T, Humbert JC, De Talance N, Buisine J. Leukocyte, lymphocyte and platelet response to dynamic exercise. Duration or intensity effect? Eur J Appl Physiol 1986; 55: 456-70.

12 Gimenez M, Mohan-Kumar T, Humbert JC, De Talance N, Teboul M, Belenguer EJA. Training and leucocyte, lymphocyte and platelet response to dynamic exercise. J Sport Med 1987; 27: 172-7.

13 Peatfield RC, Gawel MJ, Clifford-Rose F, Guthrie DL, Pearson TC. The effects of exercise on platelet numbers and size. Med Lab Sci 1985; 42: 40-3.

14 McCarthy D, Dale MM. The leucocytosis of exercise: a review and model. Sports Med 1988; 6: 333-6.

15 Groner W. New developments in flow cytochemistry technology. Proceedings of the H-1 Technicon haematology Symposium. USA: Technicon, 1987.

16 Weng B, Ramirez MA, Burns ER. The H-1 haematology analyzer. Its performance characteristics and value in the diagnosis of infectious disease. Arch Pathol Lab Med 1987; 111: 521-4.

17 Masuhara M, Kami K, Umobayashi K, Tatsumi N. Influences of exercise on leucocyte count and size. J Sports Med 1987; 27: 285-90. 